\title{
Segmentation of Brain Subjects in MR Images using Hybrid Segmentation Technique
}

\author{
P. Rajesh kumar, T. Arun Prasath, M. Pallikonda Rajasekaran, G. Vishnuvarathanan
}

\begin{abstract}
Image segmentation takes place a vital role in the area of biomedical applications. Magnetic resonance brain images with and without Alzheimer's disease have been preferred for the detection and staging the AD. Clustering is one of the extensively implemented image segmentation principle which differentiates group in such a way that samples of the relevant group are related to each other than samples associated to various groups. There has been significant concern recently in the utilization of fuzzy clustering methods, which keep additional information from the input image than the clustering principle. Modified Fuzzy C Means (MFCM) algorithm is extensively preferable because of its flexibility which leads the pixels to exist to various classes with changing the degrees of membership. Cluster initialization process has been done with MFCM and the performance of the segmentation algorithm has enhanced with Binary Gravitational search algorithm. Various brain subjects such as White Matter (WM), Grey matter (GM), hippocampus region, Cerebrospinal Fluid (CSF) are segmented for the detection of AD. The BGSA with MFCM algorithm has achieved better outcomes and it is compared with various existing techniques. The accuracy of the proposed technique is about 93.3\%.
\end{abstract}

Keywords : Alzheimer's disease, Fuzzy C Means, Gravitational search algorithm, Magnetic Resonance Imaging

\section{INTRODUCTION}

Alzheimer's Disease (AD) is the neuro degenerative brain disorder in which the brain cells starts to lose its storage capability over some course of time. The very important reason for $\mathrm{AD}$ is build-up of two proteins, called beta-amyloid and tau. Most of the Research demonstrates that these both proteins take place an important role in disease progress, but the exact reason for the AD is still not identified. [6] When the disease starts its progress, all the nerves and brain cells start damage over a time period. This damage of nerve cells leads a human to the beginning symptoms of Alzheimer's. [7] It still needs to take more concern to learn about why both of these proteins build up in the brain and in which manner it affects nerve cells of the brain. Many research are undergoing for deeper understand more about the things happen in brain tissues during Alzheimer's. Automated methods for the detection and

Revised Manuscript Received on December 16, 2019.

P.Rajesh kumar *, School of Electronics and Electrical Technology Kalasalingam Academy of Research and Education, Krishnankoil, India. Email: rkrrajesh74@gmail.com

T.Arun Prasath, School of Bio and Chemical Engineering Kalasalingam Academy of Research and Education Krishnankoil, India. Email: arun.aklu@gmail.com

M.Pallikonda Rajasekaran, School of Electronics and electrical TechnologyKalasalingam Academy of Research and Education Krishnankoil, India. Email: m.p.raja@klu.ac.in

G.Vishnuvarathanan, School of Bio and Chemical Engineering Kalasalingam Academy of Research and EducationKrishnankoil, India. Email: gvvarthanan@gmail.com classification of $\mathrm{AD}$ provides more accurate results. [10] Over the past decade numerous automated detection methods have been proposed. Among them few of the widely preferred modalities MRI produces better visualization in the detection of $\mathrm{AD}$ are functional and structural Magnetic Resonance Imaging. Few researchers have been worked on the combination of various modalities with biomarkers to improve the classification accuracy [12]. Structural based MRI AD classification principles have been followed the combination of one or more features, voxel based, and hippocampal size and cortical thickness. Hippocampus based methods follow the size and volume based measurement. Various brain matters are segmented using BGSA with MFCM algorithm for predicting the Alzheimer's disease in its very earlier stage. Cluster centroid has been initiated with MFCM algorithm as well as four different labels are declared for grouping the brain subjects.[11] In 2016 Mingxia Liu, Daoqiang Zhang et al, were worked in morphometric pattern analysis based on multi-templates for multi-view of the morphological patterns are generated from number of templates has chosen as feature representation to the MR images. An intrinsic structure-based multi-view predilection method using multiple templates to classify the AD/MCI. In order to choose multi-view feature portrayal to all the subjects using multiple chosen templates and then the operation of clustering has performed in the selected region for grouping the similar property of pixels in an individual group. Then, we determine those subclasses with exclusive codes by taking into consideration both their original own information and the class information of distribution. In [2] Yafeng Zhan, Hongxiang Yao et al were used a network-based statistic (NBS) technique for developing the cluster based structure of connected regions in AD/MCI. More number of affected subjects were analyzed and the stable and progressive AD has been idententified exactly. Most of the subjects are in the level of progressive AD after watching their behavior after few years. The outcomes provide that the subject had lower functional connectivity which can strength is being strength few functional components. Stages of AD has been calculated exactly from the connected components in the fMRI that has grouped necessary brain matters. [1] M.A.Iftikhar et al have proposed ensemble based AD classification method. Various stages of $\mathrm{AD}$ have been differentiated using ensemble of classifier. Different types of features have preferred to feed input to the classifier and those features were very helpful to enhance the accuracy. Grey matter reduction and the size shrinkage in the hippocampus are the preferred features in the classification analysis. In the stage classification analysis pMCI and sMCI have differentiated using the structural variation and the volumetric analysis.

\section{Published By:}

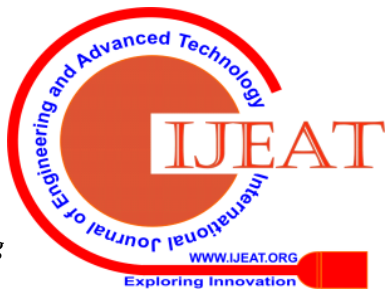




\section{Segmentation of Brain Subjects in MR Images using Hybrid Segmentation Technique}

\section{METHODOLOGY}

\section{A. Modified Fuzzy C Means}

The modified FCM principle is implemented for grouping the various types of tissues. The segmented results are evaluated depending upon the convergence rate and efficiency of segmentation. The performance of modified FCM algorithm is compared with the traditional FCM and proved that proposed algorithm has provided better results. [5] In MFCM algorithm, the distance between the centroid and the data point is calculated using mahalanobis distance. The distance measurement of data point represented by $\mathrm{M}$ and the distribution is represented as p. This is also mentioned as distance of the particular test point $p$ from the center of mass $q$ which is divided by calculated ellipsoid width (1).

$$
D(p, q)=\sqrt{(p-q)^{T} C^{-1}(p-q)}
$$

$\mathrm{C}$ is covariance matrix of the vector point $\mathrm{p}$. The distance between cluster member $x_{j}$ and the cluster center $c_{i}$ associating fuzzy logic which is characterized as (2),(3),(4)

$$
\begin{aligned}
& d^{2}\left(x_{j}, c_{i}\right)=\left(x_{j}-c_{i}\right)^{T} c_{i}\left(x_{j}-c_{i}\right) \\
& c_{i}=\left.\left|\sum_{i}\right|\right|^{1 / p}\left|\sum_{i}\right| \\
& \sum_{i}=\sum_{i=1}^{m e} u_{i, j}^{m}\left(x_{j}-c_{i}\right)\left(x_{j}-c_{i}\right)^{T} / \sum_{j=1}^{n c} u_{j=1}^{m}
\end{aligned}
$$

The distance between cluster member $x_{j}$ and the cluster center $c_{\mathfrak{i}}$ associating fuzzy logic which is characterized as (2),(3),(4)

\section{B. Binary GSA}

This paper presents the binary version of GSA [4], In order to produce this optimization technique, some of the basic principles have been modified for the implementation of this algorithm from GSA. In discrete binary domain all the dimensions can be taken 0 and 1 . The dimension changing represents the equivalent value of variable changes from 0 to $1 \mathrm{o}$ from 1 to 0 . The purpose of introducing the binary mode to initialize the gravitational search algorithm for the selection of cluster center in better manner. The updating of velocity, acceleration and the force must be assumed similarly like the continuous. The major difference between GSA and continuous algorithm is that the position updating process switching between 0 and 1 value. The switching process must be depending upon the mass velocity. In this research work, the position updating process is in the way of recently calculated bit value is the transformation with a probability function which have been measured the velocity of the mass. [3] In BGSA the velocity updating process is performed based on (eqn 6) and the recent position should be selected either 1 or 0 from the chosen probability. The velocity total large value must produce a maximum probability in the position of changing mass with corresponding existing position. The smaller value of velocity should produce a small probability value when position variation in the mass is preferable at the velocity reaches zero. Let us assume the system which is having ' $N$ ' agents (masses) and $i^{\text {th }}$ agent's position is represented by (5)

$$
X_{i}=\left(x_{i, \ldots \ldots s}^{1} x_{i, \ldots \ldots,}^{d} x_{i}^{n}\right) \text { for } i=1,2 \ldots \ldots N
$$

$x_{i}^{d}$-denoted $i^{\text {th }}$ agent's position $d^{\text {th }}$ dimension in search space dimension $\mathrm{n}$. In a specific time $\mathrm{t}$, force is acting on mass $i$ according to the mass $j$ has denoted

$$
F_{i j}^{d}(t)=G(t) \frac{M_{p^{i}}\left[(t) \times M_{a j}[t]\right.}{R_{i j}(t)+\varepsilon}\left(x_{j}^{d}-x_{i}^{d}(t)\right)
$$

The total force acting on the agent in dimension $\mathrm{d}$ and the $k_{\text {best }}$ agent is calculated by the below formula (6)(7).

$$
F_{i}^{t}=\sum_{j \in h b s t, j \neq i} \operatorname{rand}_{j} F_{i j}^{d}(t)
$$

According to the law of motion the agent $i$ acceleration in time $t$ in direction $\mathrm{d}$ and $a_{\mathrm{i}}^{d}(t)$ is defined as (8)

$$
a_{i}^{t}=\frac{F_{i}^{a}(t)}{M_{i i}(t)}
$$

Where the agent $i^{\text {th }}$ mass inertia is $M_{\text {ii }}$ the next velocity of the particular agent is calculated as the fraction of that current velocity is added with its acceleration. The position and the velocity of the agent (9),(10) can be written as

$$
\begin{gathered}
v_{i}^{d}(t+1)=\operatorname{rand}_{i} \times v_{i}^{d}(t)+a_{i}^{d}(t) \\
x_{i}^{d}(t+1)=x_{i}^{d}(t)+v_{i}^{d}(t+1)
\end{gathered}
$$

Initial and fractional masses are measured with the help of fitness evaluation more efficient agent is possible with heavier mass which means that the particular agent is having higher attraction and it moves slowly(11),(12).

$$
\begin{aligned}
& q_{i}(t)=\frac{f_{i t}(t) \text {-worgt }\left(t_{i}\right)}{\text { best }\left(t^{j}\right) \text {-worst }(t)} \\
& M_{i}(t)=\frac{q_{i}(t)}{\sum_{j=1}^{N} q_{j}(t)}
\end{aligned}
$$

where $f i t_{i}(t)$ mentions the best fitness value of the agent $i$ at the particular time $t$ and best $(t)$ and worst $(t)$ are defined as (13),(14).

$$
\begin{aligned}
& \text { best }(t)=\min _{j \in\left[1_{1} m\right]} f i t_{j}(t) \\
& \text { worst }(t)=\min _{j \in\left[1_{1} m \mathbb{N}\right.} f i t_{j}(t)
\end{aligned}
$$

\section{Feature selection}

Feature selection is an essential task in medical imaging analysis and classification process. Features have been taken using Grey Level Co-occurrence Matrix principle. Additionally the cortical thickness, size of the hippocampus volume of GM, WM have been included for the better performance of the classifier.

\section{RESULT AND DISCUSSION}

For this research work 200 brain AD images have been obtained from OASIS (Open Access Series of Imaging Studies) https://www.oasis-brains.org/. MRI T1 weighted noiseless images have been taken and used 120 images for training and 80 images for testing. Figure 1.(a) is axial input image with skull region, generally skull region affects the segmentation accuracy. In order rectify this issues, Skull stripping process has been done with brain extraction tool. Figure 1.(b) shows the brain region after the skull stripping. Figure 1.(c) represents the normalized image. [8] Normalization tasks is very useful to enhance the pixel intensity which increases the segmentation accuracy. Figure 1.(d) denotes the segmented outcomes of K-Means clustering algorithm. Three different types of clusters were assigned to segment the WM, GM hippocampus region, CSF. Various cluster initialization strategies have been adopted with different protocols. Figure 1.(e) shows the output of PSO-K means clustering algorithm which gives the better results than the $\mathrm{k}$ mean clustering algorithm. All the brain subjects were segmented more accurately using PSO K means principle [9]. Cluster initialization has performed with random manner. Then the results are optimized using PSO. Figure 1.(f) denotes the result of proposed BGSA with MFCM algorithm. FCM algorithm which is modified slightly in the membership function. This algorithm has given better results than other existing algorithm. The Above 
stated strategies have been implemented Figure 2.(a)-6.(a).

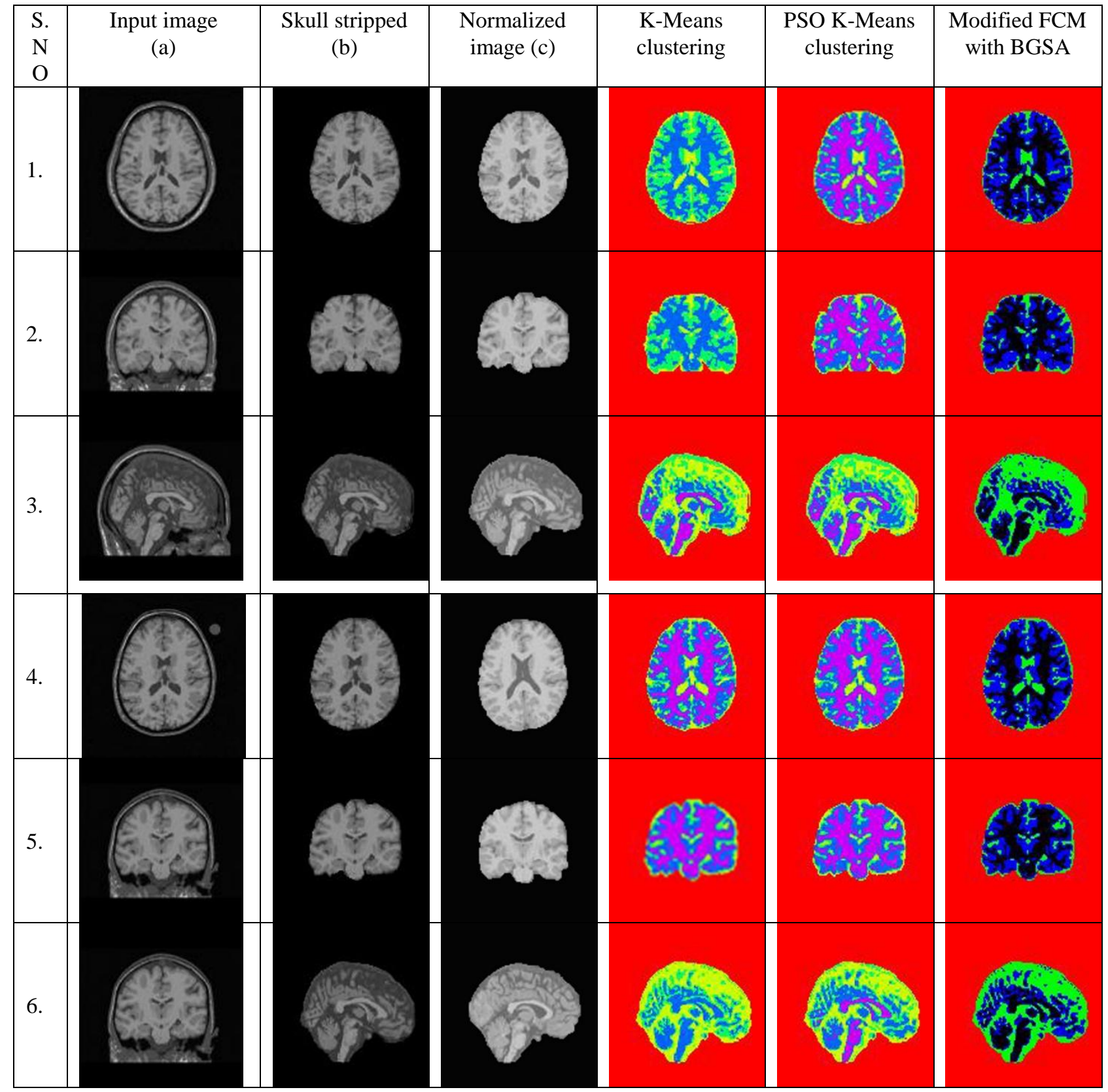

Figure 1. Results of MFCM with BGSA algorithm

1.1(a)-6.1(a)-Input images, 1.1(b)-6.1(b)- skull stripped images, 1.1(c)-6.1(c)- Normalized images , 1.1(d)-6.1(d)Segmentation result of K-Means algorithm, 1.1(e)-6.1(e)- segmentation results of PSO K Means algorithm, 1.1(f)-6.1(f)segmentation output of MFCM with BGSA algorithm

\begin{tabular}{|c|c|c|c|c|}
\hline Image & Type & Accuracy & Specificity & Sensitivity \\
\hline \multirow{3}{*}{1} & Coronal & 0.8453 & 0.1282 & 0.8250 \\
\cline { 2 - 5 } & Axial & 0.8568 & 0.1282 & 0.8290 \\
\hline \multirow{2}{*}{2} & Sagittal & 0.8789 & 0.1282 & 0.8390 \\
\cline { 2 - 5 } & Coronal & 0.8123 & 0.1282 & 0.8332 \\
\cline { 2 - 5 } & Axial & 0.8143 & 0.1282 & 0.8465 \\
\hline
\end{tabular}

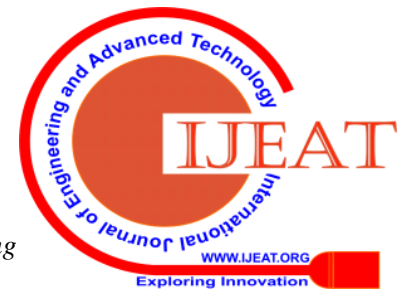




\section{Segmentation of Brain Subjects in MR Images using Hybrid Segmentation Technique}

\begin{tabular}{|c|c|c|c|c|}
\hline \multirow{3}{*}{3} & Coronal & 0.8980 & 0.1282 & 0.8876 \\
\hline & Axial & 0.8645 & 0.1282 & 0.8712 \\
\hline & Sagittal & 0.8334 & 0.1282 & 0.8432 \\
\hline \multirow{3}{*}{4} & Coronal & 0.8876 & 0.1282 & 0.8564 \\
\hline & Axial & 0.8897 & 0.1605 & 0.8675 \\
\hline & Sagittal & 0.8534 & 0.1500 & 0.8762 \\
\hline \multirow{3}{*}{5} & Coronal & 0.8723 & 0.1282 & 0.8772 \\
\hline & Axial & 0.8426 & 0.1282 & 0.8987 \\
\hline & Sagittal & 0.8768 & 0.1282 & 0.8897 \\
\hline \multirow{3}{*}{6} & Coronal & 0.8542 & 0.1282 & 0.8709 \\
\hline & Axial & 0.8443 & 0.1282 & 0.8930 \\
\hline & Sagittal & 0.8329 & 0.1282 & 0.8895 \\
\hline \multirow{3}{*}{7} & Coronal & 0.8893 & 0.1282 & 0.8881 \\
\hline & Axial & 0.8426 & 0.1282 & 0.8645 \\
\hline & Sagittal & 0.8820 & 0.1282 & 0.8587 \\
\hline \multirow{3}{*}{8} & Coronal & 0.8650 & 0.1282 & 0.8234 \\
\hline & Axial & 0.8980 & 0.1282 & 0.8712 \\
\hline & Sagittal & 0.8270 & 0.1282 & 0.8709 \\
\hline \multirow{3}{*}{9} & Coronal & 0.8341 & 0.1282 & 0.8996 \\
\hline & Axial & 0.8687 & 0.1282 & 0.8325 \\
\hline & Sagittal & 0.8826 & 0.1282 & 0.8334 \\
\hline \multirow{3}{*}{10} & Coronal & 0.8754 & 0.1282 & 0.8824 \\
\hline & Axial & 0.8967 & 0.1282 & 0.8676 \\
\hline & Sagittal & 0.8978 & 0.1282 & 0.8744 \\
\hline
\end{tabular}

Table 1: Evaluation parameters for segmentation

Table 1 represents the evaluation parameters for prove the accuracy of the proposed segmentation algorithm. The proposed algorithm has achieved better results when compared with the existing research work.

\section{Conclusion}

This research work focused on the brain subject segmentation using MFCM with BGSA algorithm. Various brain matters are clustered for the detection of AD. Volumetric analysis of WM, GM, Hippocampus region which helps to predict the AD accurately in its very earlier stage. BSA algorithm is adopted after the segmentation, in order to improve the performance of FCM algorithm. This process is essential in the pre-surgical planning. The future works will be on feature selection and classification of AD.

Acknowledgement:

The authors would like to thank the management of kalasalingam academy of research and education and the school of electronic and electrical technology for providing the computational facilities in VLSI research laboratory.

\section{REFERENCES}

[1] Iftikhar MA, Idris A. An ensemble classification approach for automated diagnosis of Alzheimer's disease and mild cognitive impairment. In2016 International Conference on Open Source Systems \& Technologies (ICOSST) 2016 Dec 15 (pp. 78-83). IEEE.

[2] Zhan, Y., Yao, H., Wang, P., Zhou, B., Zhang, Z., Guo, Y.E., An, N., Ma, J., Zhang, X. and Liu, Y., 2016. Network-Based Statistic Show Aberrant Functional Connectivity in Alzheimer's Disease. IEEE Journal of Selected Topics in Signal Processing, 10(7), pp.1182-1188.

[3] Rashedi, Esmat, Hossein Nezamabadi-Pour, and Saeid Saryazdi. "BGSA: binary gravitational search algorithm." Natural Computing 9 , no. 3 (2010): 727-745

[4] Hooda, Heena, Om Prakash Verma, and Sonam Arora. "Optimal Fuzzy C-Means Algorithm for Brain Image Segmentation." In Applications of Artificial Intelligence Techniques in Engineering, pp. 591-602. Springer, Singapore, 2019.

[5] Valverde, Sergi, Arnau Oliver, Eloy Roura, Sandra González-Villà, Deborah Pareto, Joan C. Vilanova, Lluís Ramió-Torrentà, Àlex Rovira, and Xavier Lladó. "Automated tissue segmentation of MR brain images in the presence of white matter lesions." Medical image analysis 35 (2017): 446-457.

[6] Shanthi, K. J., D. K. Ravish, and M. Sasikumar. "Image segmentation an early detection to Alzheimer's disease." In 2013 Annual IEEE India Conference (INDICON), pp. 1-4. IEEE, 2013.

[7] Kumar, P. Rajesh, Thiyagarajan Arunprasath, M. Pallikonda Rajasekaran, and G. Vishnuvarthanan. "Computer-aided automated discrimination of Alzheimer's disease and its clinical progression in magnetic resonance images using hybrid clustering and game theory-based classification strategies." Computers \& Electrical Engineering 72 (2018): 283-295.

Published By: 
[8] Kumar, P. Rajesh, et al. "Brain Subject Estimation Using PSO K-Means Clustering-An Automated Aid for the Assessment of Clinical Dementia." International Conference on Information and Communication Technology for Intelligent Systems. Springer, Cham, 2017.

[9] Chatterjee, Priyam, and Peyman Milanfar. "Patch-based near-optimal image denoising." IEEE Transactions on Image Processing 21, no. 4 (2011): 1635-1649.

[10] Coupé, Pierrick, José V. Manjón, Vladimir Fonov, Jens Pruessner, Montserrat Robles, and D. Louis Collins. "Patch-based segmentation using expert priors: Application to hippocampus and ventricle segmentation." NeuroImage 54, no. 2 (2011): 940-954.

[11] Liu, Mingxia, Daoqiang Zhang, and Dinggang Shen. "Relationship induced multi-template learning for diagnosis of Alzheimer's disease and mild cognitive impairment." IEEE transactions on medical imaging 35, no. 6 (2016): 1463-1474.

[12] Rousseau, Françcois, Piotr A. Habas, and Colin Studholme. "A supervised patch-based approach for human brain labeling." IEEE transactions on medical imaging 30, no. 10 (2011): 1852-1862. 